Revue d'histoire des chemins de fer

\section{Revue d'histoire des chemins de fer}

$42-43 \mid 2012$

"Vitesse ferroviaire, gestion du temps et construction des territoires" et articles divers

\title{
Le Train-Ferry Douvres-Dunkerque, le Train Bleu et autres initiatives intermodales dans la France de l'entre-deux-guerres
}

The Train-Ferry between Dover and Dunkirk, Le Train Bleu and Other

Intermodal Initiatives in Interwar France

\section{Natalia Starostina}

Traducteur : Christine Cross

\section{OpenEdition \\ Journals}

Édition électronique

URL : https://journals.openedition.org/rhcf/1524

DOI : 10.4000/rhcf.1524

Éditeur

Rails \& histoire

Édition imprimée

Date de publication : 1 septembre 2012

Pagination : 49-65

ISSN : 0996-9403

\section{Référence électronique}

Natalia Starostina, «Le Train-Ferry Douvres-Dunkerque, le Train Bleu et autres initiatives intermodales dans la France de l'entre-deux-guerres », Revue d'histoire des chemins de fer [En ligne], 42-43 | 2012, mis en ligne le 14 novembre 2014, consulté le 22 avril 2022. URL : http://journals.openedition.org/rhcf/ 1524 ; DOI : https://doi.org/10.4000/rhcf.1524 
Natalia STAROSTINA

\title{
Le Train-Ferry Douvres-Dunkerque, le Train Bleu et autres initiatives intermodales dans la France de l'entre-deux-guerres
}

\author{
Traduit de l'américain par Christine Cross
}

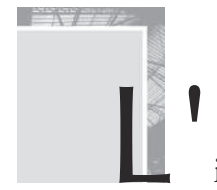

intermodalité ${ }^{*}$ c'est-à-dire le concept du voyage sans rupture de charge, n'est autre qu'une manifestation du désir fondamental de l'être humain de pouvoir se déplacer en dehors de toute contrainte imposée par les frontières créées par la nature ou par l'homme. L'intermodalité est l'un des facteurs déterminants dans le façonnage de nos cultures de mobilité moderne : à l'heure de la mondialisation tous azimuts, rêver de parcourir le monde sans solution de continuité est probablement gravé dans notre inconscient contemporain ${ }^{1}$. À une époque où le nombre de voyageurs ne cesse de monter en flèche et où les distances parcourues connaissent une évolution non moins radicale, les

\footnotetext{
* Je profite de l'occasion pour remercier très sincèrement Michèle Merger de m'avoir invitée à participer au colloque COST 340 en France. L'invitation de l'AHICF et l'accueil chaleureux qui m’a été réservé m'ont inspirée à plusieurs titres ; par son organisation exceptionnelle et par le niveau de ses échanges, ce colloque fait partie des événements les plus fructueux et les plus riches en enseignements auxquels j’ai pu assister. Je remercie également Christian Fonnet, chargé du fonds documentaire de l'AHICF, qui m'a fait connaître les études les plus récentes en la matière. Je voudrais enfin saluer les Professeurs Lee March, Ron Roach, et Cathy Cox (Young Harris College) qui ne m'ont pas simplement encouragée et soutenue moralement, mais m'ont également aidée financièrement, entre autres choses, en mettant à ma disposition les fonds nécessaires à mon voyage d'étude de fin de semestre à Paris.

1- Sur le concept de la mobilité, voir FUMEY, VARLET, ZEMBRI (sous la dir.), 2009.
} 
réseaux de transport intermodal sont à l'image des ambitions premières de l'homme, qui souhaite pouvoir rayonner à travers la planète, rapidement et en toute simplicité : aujourd'hui, voyager symbolise le triomphe de la technologie sur le temps et sur l'espace. Le colloque final de l'Action COST 340 sur l'intermodalité tenu à Paris en 2005 a réuni des représentants des milieux politique, industriel et universitaire venus aborder la problématique de l'intermodalité et tirer les enseignements du passé.

Même si, pour beaucoup d'historiens, l'apparition des voyages intermodaux remonte à la période d'après guerre, les premières initiatives européennes datent, en fait, du XIx ${ }^{e}$ siècle, la plus légendaire étant probablement le célèbre Orient-Express. Pendant les années qui ont séparé les deux guerres, les compagnies de chemins de fer françaises ont lancé bon nombre de projets en vue d'améliorer les liaisons entre la France, l'Angleterre et le Nouveau Monde. Le Train Bleu, ce train de luxe qui acheminait l'aristocratie britannique vers les stations balnéaires les plus prisées de la Côte d'Azur, constituait un bel exemple de l'intermodalité au service d'une clientèle franco-britannique fortunée. Voyages, la revue mensuelle illustrée de la Compagnie Paris-LyonMéditerranée (PLM), mettait en exergue le confort inégalé du Train Bleu², les passagers voyageant de Londres à Paris et au-delà jusqu'à la Côte d'Azur, grâce à un ferry et à un train confortablement aménagé.

Le projet intermodal le plus insolite de la période de l'entre-deuxguerres a vu le jour à Dunkerque. Inauguré en 1936, le Night Ferry est né de la collaboration entre différentes compagnies de transport ferroviaire et maritime françaises et britanniques. Grâce à lui, les passagers français et britanniques pouvaient effectuer leur déplacement en train dans des conditions agréables : le soir ils prenaient place dans le train à Londres et le lendemain matin ils se réveillaient à Paris, le train ayant emprunté un ferry-boat pour traverser la Manche, avant de poursuivre leur voyage sur le continent européen. D’une longévité remarquable, le Night Ferry n'a cessé de circuler qu'en 1980. Son image, relayée par les revues illustrées publiées en France et en Angleterre, mettait l'accent sur le niveau de confort hors pair du voyage à son bord; en empruntant le Night Ferry les voyageurs pouvaient dormir paisiblement sur tout le parcours. Les affiches renvoyaient aussi une image forte de la collaboration entre acteurs français et britanniques dans la mise en place de cette solution intermodale aussi merveilleuse qu'inédite.

2- Voyages. Revue mensuelle illustrée, SNCF, Centre des archives historiques, Le Mans (désormais : CAH), B.C.P. $56,57,57$. 
Un autre facteur important dans le présent article concerne la manière dont ces initiatives intermodales ont été représentées dans la presse française. Bon nombre de photos et d'articles traitant des nouvelles initiatives intermodales, parmi elles le train-ferry et le Train Bleu, ont paru dans les revues illustrées et autres publications parrainées par les compagnies de chemin de fer françaises (précisons que la publicité ferroviaire a connu un grand essor pendant la Grande Dépression). Conscientes de la baisse intervenue dans le nombre de visiteurs étrangers, plusieurs compagnies ont soit créé un service de publicité soit procédé à l'étude en profondeur des vecteurs adoptés par les publicitaires indépendants pour la présentation de leurs services. C'est à partir de 1929 que les compagnies ferroviaires ont commencé à comprendre que la publicité pouvait leur permettre de concurrencer l'avion et la voiture et d'attirer de nouveaux clients vers le rail ${ }^{3}$.

Si les initiatives prises entre les deux guerres ont connu une telle réussite, c'est non seulement parce qu'elles facilitaient le voyage et en augmentaient le confort mais également parce qu'elles étaient en concordance avec le nouveau discours sur la mobilité moderne et, au-delà, l'identité française. En mettant en avant la notion de confort inégalé ou l'amitié entre la France et la Grande-Bretagne, l'image projetée par les initiatives intermodales a su à la fois nourrir et s'inspirer de ce nouveau discours sur l'identité nationale et la perception de l'espace français. Ainsi, en chatouillant l'imagination des Français, les images en question avaient la capacité de créer de nouvelles mythologies dans les domaines du voyage et de l'espace tout en contribuant au succès des initiatives intermodales.

Durant la Belle Époque, la Compagnie internationale des wagons-lits (CIWL) a lancé quelques projets intéressants, qui ont joué un rôle précurseur en matière de réseaux intermodaux $x^{4}$. La compagnie proposait à sa clientèle aisée un niveau de confort hors du commun et la promesse de pouvoir voyager de Paris jusqu'en Russie, en Espagne voire en Turquie sans changer de train avait tout pour envoûter et fasciner la haute société européenne. En lançant les voitures Pullman et en négociant avec les compagnies de chemin de fer et les États concernés, George Nagelmackers, grand visionnaire et fondateur de

3- Voir Chemin de fer du Nord ; Centre D'instruction ; Trafic voyageurs au point de vue commercial ; Tourisme-publicité-Concurrence ; Conférence faite par M. Bardin, Chef-Adjoint des Services Commerciaux (Lille, 1930-1931) : CAH, 26 J3.

4- Sur l'histoire de la compagnie, voir CIWL, 1926. Voir également COUDERT, KNEPPER TOUSSIROT, 2009 ; DES CARS, CARACALLA, 1984 ; BEHREND, 1977 ; CAMPBELL, 1983 ; COMMAULT, 1966 ; DES CARS, COMMAULT, 1976 ; COOKRIDGE, 1978 ; BEHREND, KELLY, 1969 ; SHERWOOD, 1985 ; WIESENTHAL, 1979. 
l'entreprise, a offert aux voyageurs européens fortunés le train Orient-Express. Ce train incarnait le voyage de loisir dans toute sa splendeur car il permettait de se rendre à Constantinople, destination exotique et séduisante. Le trajet comportait la traversée d'un lac en bateau : à Ruschuk, en Bulgarie, les voyageurs devaient emprunter un ferry-boat sur le parcours menant à la célèbre ville turque ${ }^{5}$. Vers la fin du XIX $x^{e}$ siècle, le nombre de trains de voyageurs rapides et confortables franchissant les frontières a connu un bond extraordinaire : il y avait des trains permettant aux touristes britanniques de rejoindre rapidement la Côte d'Azur, des trains effectuant la liaison avec les ports où l'on pouvait embarquer sur des paquebots à destination de l'Amérique du Sud, et de nombreux trains acheminant les voyageurs vers diverses destinations de l'Europe septentrionale ${ }^{6}$.

Les trains à long parcours ont vite capté l'imagination des Européens et, à bien des égards, façonné la perception du voyage en train durant la Belle Époque. Sous la plume des écrivains et des poètes, les « trains de luxe » étaient généralement associés au prestige et à des prestations haut-de-gamme. Le poète français Valéry Larbaud a même écrit un poème consacré à l'Orient-Express:

"Prête-moi ton grand bruit, ta grande allure si douce,

Ton glissement nocturne à travers l'Europe illuminée,

Ô train de luxe! Et l'angoissante musique

Qui bruit le long de tes couloirs de cuir doré,

Tandis que derrière les portes laquées, aux loquets de cuivre lourd,

Dorment les millionnaires.

Je parcours en chantonnant tes couloirs

Et je suis ta course vers Vienne et Budapest,

Mêlant ma voix à tes cent mille voix,

Ô Harmonika-Zug ${ }^{7} !(\ldots)$ »

Sans aller jusqu'à décrire ses passagers, le poète brosse par ces vers un portrait éloquent de l'univers du pouvoir et de l'argent évoqué par l'OrientExpress. L'écrivain Paul Morand a même fait de ce train l'un des symboles de la Belle Époque ${ }^{8}$. Issu d'une famille aristocratique, Paul Morand bénéficiait du soutien de Philippe Berthelot, fonctionnaire haut placé au sein du ministère des Affaires étrangères'. Selon Morand, si l'image des riches dépeinte dans les

\footnotetext{
5- GREGORY, 1991, p. 119.

6- Pour une description de ces trains, on peut utilement recourir à l'œuvre de François Caron ainsi qu'à l'histoire de la CIWL : BACHET, BALSO, BEJUI, et al., 1999, p. 269-271.

7- Cité in GREGORY, 1991, p. 116.

8-Sur le discours surl'Orient-Express dans la France de l'entre-deux-guerres, voir STAROSTINA, 2007, p. 153-227.

9- Voir GUITARD-AUVISTE, 1981 et CRONIN, 1994, p. 25-29.
} 
romans de Claudel devait correspondre à la réalité et si les gens richissimes devaient vraiment remplir leurs poches et leurs valises de diamants et de billets de banque, alors ce serait à bord de l'Orient-Express, "le train des milliardaires $»^{10}$, qu'on pourrait croiser de telles personnes. Pour Morand, l'achat d'un billet pour l'Orient Express donnait un accès automatique au monde des élites. Selon son expression, l'Orient-Express était " le vrai cordon ombilical " ${ }^{11}$ reliant l'élite aristocratique et la bourgeoisie à travers le monde. Ainsi, durant la Belle Époque, les réseaux de transport intermodal évoquaient le luxe mais ils se distinguaient aussi et surtout, au cours des années précédant la Première Guerre mondiale, par l'accent mis sur le caractère exclusif et élitiste de ces trains.

Dès le début de la Grande Guerre, plusieurs trains de luxe long parcours ont cessé de circuler et parmi eux l'Orient-Express. De surcroît, pour satisfaire les besoins des forces armées, les wagons-restaurants de la CIWL ont été réquisitionnés pour en faire des voitures sanitaires ${ }^{12}$. Dans le même temps, les forces armées ont également tenté de relier différents modes de transport entre eux. Tel fut, par exemple, le cas des ferry-boats mis en service à travers la Manche vers la fin de la guerre. L'armée britannique avait lancé deux trains-ferrys, le premier entre Harwich et Dunkerque, le second (inauguré le 22 février 1918) entre Newhaven et Dieppe ${ }^{13}$. De telles initiatives visaient essentiellement le transport de marchandises.

Les affiches publicitaires des chemins de fer ont joué un rôle important dans la promotion de l'amitié entre la France, la Grande-Bretagne et les ÉtatsUnis. Pendant les décennies qui ont séparé les deux guerres, les relations entre la France et la Grande-Bretagne n'ont pas toujours été au beau fixe : l'une des principales sources de tensions entre les deux pays résidait dans le fait que l'élite politique en France était résolue à isoler l'Allemagne et à lui faire payer la mise à sac du nord de la France tandis que les Britanniques, au contraire, préféraient favoriser sa reconstruction et son intégration au sein de l'Europe. À la même époque, dans le domaine des échanges touristiques, les compagnies de chemin de fer françaises et l'office du tourisme essaient d'attirer un maximum de visiteurs britanniques. Il s'agissait, avant tout, d'inciter les ressortissants britanniques fortunés à visiter Paris et à passer l'hiver sur la Côte d'Azur. Et, pour améliorer les communications entre la France, la Grande-Bretagne,

10- MORAND, 1994, p. 45-56, citation p. 52.

11- Ibid.

12- COUDERT, KNEPPER, TOUSSIROT, 2009, p. 32.

13- L'Illustration, 2 mars 1918, cité in «Le nouveau service de ferry-boats de Dunkerque à Douvres », idem, 24 octobre 1936. 
l'Amérique du Nord et l'Amérique du Sud, la direction des chemins de fer lança diverses initiatives intermodales dans le nord-ouest de la France.

Pour offrir aux classes moyennes françaises et européennes la possibilité d'accéder à de nouvelles destinations de loisirs, la CIWL et les compagnies de chemins de fer françaises avaient lancé, dès 1922, le Calais-MéditerranéeExpress (ou Train Bleu) en direction de la Côte d'Azur. L'entreprise ferroviaire française assurait la correspondance entre le Train Bleu et un train britannique partant de Londres-Victoria ${ }^{14}$. Une rame française attendait les touristes à Calais pour les acheminer vers la gare de Lyon puis en direction de Nice, où ils devaient arriver dès le lendemain matin. En une seule nuit, ils étaient transportés par le rail jusqu'à la Côte d'Azur, pays de soleil et de chaleur ${ }^{15}$. La CIWL proposait de nouvelles voitures-lits en acier, plus stables (chacune ayant un poids de 53 tonnes) et plus confortables que les anciennes voitures ${ }^{16}$. En dépit de ces nouveaux matériels, la direction de la CIWL a tenu absolument à préserver à bord du Train Bleu la qualité de service légendaire des trains de luxe. Le numéro spécial du cinquantenaire de la CIWL définissait le Train Bleu comme représentant « la quintessence du luxe, de la sécurité et du confort ${ }^{17}$.

Pendant les années 1920, les compagnies de chemin de fer françaises et la CIWL ont tout mis en œuvre pour entretenir l'image du Train Bleu en tant que moyen de transport de prédilection des vacanciers aisés, les différentes publications étant censées inciter les visiteurs venus d'ailleurs à emprunter le train pour rejoindre la Côte d'Azur et ainsi profiter du soleil, même en plein hiver $^{18}$. La brochure intitulée Swift Services to the South ${ }^{19}$, dédiée à un public anglophone, présentait les différentes options disponibles pour se déplacer entre Londres et Paris en première classe, citant particulièrement le Train Bleu.

14- «Les deux premiers “Trains Bleus” », L'Éclaireur de Nice, 10 décembre 1922.

15- Pour tous les détails concernant l'histoire du Train Bleu, voir DES CARS, CARACALLA, 1988.

16- CIWL, 1926, p. 28.

17- Ibid., p. 16.

18- Par exemple, "Europeans Find Fault with our Sleeping Cars: Lack of Privacy Annoys Them » [Les Européens critiquent nos voitures-lits : le manque d'intimité les dérange], Chicago Daily Tribune 1928 ; « 2 Ways to Winter Sunshine » [2 moyens pour atteindre le soleil en hiver], The Times, 1926 ; «Famous British Expresses: A Link with the Continent " [Trains-express anglais de renom : une liaison avec l'Europe continentale], The Times, 1927 ; «The French Riviera: Colour, Gaiety, and Showmanship: From Our Riviera Correspondent » [La Côte d'Azur : de la couleur, de la joie et du spectacle - lettre de notre correspondant], The Times, 1937.

19- Swift Services to the South [Services rapides à destination du Sud], Paris, 1938. 
Le Chicago Tribune dépeignait le Train Bleu comme étant le symbole de la prospérité et de la richesse ${ }^{20}$.

Le Train Bleu attirait de nombreux voyageurs britanniques et américains : pour le gratin de la société anglo-saxonne, il est devenu un produit très convoité, voire l'une des grandes modes des années 1920. À en croire les petites annonces publiées dans le Times, toutes les places disponibles étaient réservées plusieurs semaines à l'avance au point que, quels que soient leurs moyens, les personnes souhaitant voyager à bord du train avaient beaucoup de mal à se procurer des billets. En 1924, un dénommé McGowan, qui cherchait désespérément deux places dans le Train Bleu pour se rendre à Cannes dans les premiers jours de février, fit publier une petite annonce en ce sens. Dans une autre annonce datée de 1923, une dame anglaise proposait deux places dans sa Rolls Royce au "tarif Train Bleu " à des personnes voulant se rendre sur la Côte d'Azur. Les articles de la presse faisaient état de ces difficultés dans la réservation de billets pour le Train Bleu au début des années 1920, plusieurs années plus tard on relatait encore les déboires de ces dames qui avaient dû prolonger leur séjour sur la Côte d'Azur faute de places dans le train de leurs rêves. Dans la rubrique "Courrier des lecteurs " du Times, on trouve encore des propos élogieux à l'égard du Train Bleu et de la simplicité des procédures de douane qui lui sont réservées ${ }^{21}$. Enfin, à plusieurs reprises au cours des années 1920 et 1930, le train a figuré dans les colonnes du quotidien américain le Chicago Daily Tribune 22 .

20- Il n'existe probablement pas de documents écrits permettant de confirmer la rumeur selon laquelle, pour accroître la popularité du Train Bleu, les compagnies de chemin de fer françaises auraient payé les auteurs de tels articles.

21- W. St.D. Jenkins, "The Channel Crossing: To the Editor of the Times » [Traversée de la Manche : Lettre au Rédacteur en chef], The Times, 1926, lettre datée du 25 janvier. M. Jenkins précisait qu'il appartenait au New University Club.

22- Voir par exemple «France plans to soak tourist travel from the U.S. » [La France compte écrémer les touristes en provenance des États-Unis], Chicago Daily Tribune, 7 février 1924 ; Pamela Frankan, «Twin in Search of a Shirt: Life on the Riviera Made Evelyn Lennox Think of Champagne Cocktails, Baccarat, and Women in Love ; His Presence There Almost Added a Violent and Incongruous Note to the Picture » Jumeau à la recherche d'une chemise ; pour Evelyn Lennox la vie sur la Côte d'Azur évoquait les cocktails au champagne, le jeu de baccarat et les femmes amoureuses; sa présence sur les lieux à failli donner à cette image une touche violente et incongrue], Chicago Daily Tribune, 5 juillet 1931 ; Swinnerton, «The Beauty of the Blue Train » [La Beauté du Train Bleu], Chicago Daily Tribune, 19 juin 1927, 1-3 ; « King on verge of Abdication: Cabinet acts today on farewell of Edward ; Mrs. Simpson in Cannes » [Abdication du Roi imminente : le Cabinet se prononce aujourd'hui sur le départ d'Édouard ; Mme Simpson à Cannes], Chicago Daily Tribune, 6 décembre 1936, ainsi que beaucoup d'autres articles. 
Le Train Bleu, cette liaison créée entre les deux guerres et présentant déjà une dimension intermodale, associait la notion de confort à l'ancienne à celle de confort moderne : d'un côté il s'agissait d'un train rapide et sûr, de l'autre d'une rame dont l'agencement évoquait une certaine nostalgie de la France de la Belle Époque. Mais retenons surtout que les notions d'intermodalité et de confort se sont fondues l'une dans l'autre entre les deux guerres pour forger une nouvelle culture de mobilité.

Le lancement en 1936 du train-ferry Douvres-Dunkerque a marqué l'une des principales étapes de la mise en place de réseaux de transport intermodal dans la France de l'entre-deux-guerres ${ }^{23}$. Rappelons que les passagers rejoignaient à Londres-Victoria un train qui partait en direction de Douvres où les voitures étaient chargées à bord du ferry-boat. Le lendemain matin les passagers se réveillaient à Paris. La presse vantait les mérites d'un tel voyage ininterrompu entre deux nations. Finie l'époque où les voyageurs ferroviaires devaient se réveiller au milieu de la nuit pour traverser la Manche en ferryboat. À bien des égards, le train-ferry incarnait l'aboutissement d'un rêve centenaire, celui de forger une liaison fiable entre l'Angleterre et la France.

Le principe même du train-ferry date de la fin du XIx ${ }^{e}$ siècle mais, à plusieurs reprises, le gouvernement britannique avait rejeté des projets visant la mise en place d'une telle liaison. Se fondant sur des documents soumis au Parlement lors de la tentative de 1905, Ernest de Rodakowski publia la même année une étude détaillée intitulée The Channel ferry: Advantages and feasibility of a train-ferry between England and France ${ }^{24}$. Dans ce travail, Rodakowski soulignait les nombreux avantages d'une liaison ininterrompue entre l'Angleterre et la France. Tout en évitant la double manutention des marchandises, le train-ferry devait surtout faire du déplacement entre Londres et Paris une expérience confortable et agréable. Devoir descendre du train et monter à bord d'un bateau au milieu de la nuit, et ceci quel que soit le sens du voyage, constituait selon lui " une gêne inhumaine qui, en interrompant le sommeil des passagers, ne faisait qu'aggraver considérablement la fatigue du voyage $»^{25}$. La monographie mettait surtout en évidence les avantages qui découleraient de la construction et de la mise en service d'un train-ferry entre Douvres et Calais, produisant des dessins pour illustrer la logique sous-tendant l'ingénierie du projet (une commission formée par le gouvernement avait par ailleurs,

23- LATRASSE, 1936, p. 232-241.

24- RODAKOWSKI, 1905.

25- Ibid., p. 5. 
à la même époque, envisagé la liaison entre Newhaven et Dieppe). En 1936, le train-ferry voit enfin le jour et rallie finalement Douvres à Dunkerque.

Des deux côtés de la Manche, dans les années 1930, les médias ont suivi de près la construction du train-ferry. D'après un article paru dans le Times en 1932, sa création aurait exigé la coordination des efforts du Réseau du Nord, de la chambre de commerce de Dunkerque, de Southern Railways et de la Compagnie internationale des wagons-lits ${ }^{26}$. Dans les mois qui ont précédé le lancement du train-ferry, le Times a publié des articles décrivant le processus adopté pour sa construction et nommant les acteurs impliqués dans sa réalisation. L'inauguration du train-ferry a également constitué un événement de taille pour les milieux diplomatiques. Selon le Times, elle a donné lieu à diverses réceptions et autres cérémonies auxquelles ont assisté de nombreuses personnalités publiques, aux côtés d'artisans du train-ferry tels que Sir Herbert Walker, directeur général de Southern Railways, qui a consacré d'énormes efforts au projet ${ }^{27}$. Lors du banquet officiel, il eut l'honneur d'être promu commandeur de la Légion d'Honneur.

Par ailleurs, pour les ambassadeurs britannique et français, la promotion du train-ferry a facilité celle de l'amitié entre leurs deux nations et ils ont fait de cette initiative technologique un symbole de partenariat. ${ }^{28}$ Pour les médias, le train-ferry Douvres-Dunkerque est vite devenu la manifestation extérieure de l'amitié franco-britannique, certains allant jusqu'à le comparer à un tapis magique, le qualifiant de technologie miraculeuse permettant de voyager entre les deux pays. On évoque aussi « le ferry qui confortera l'amitié entre les différents pays du monde » ou " une nouvelle solution attrayante permettant d'atteindre le centre de l'Empire, une liaison qui renforcera et qui resserrera les liens entre deux grandes nations $»^{29}$. Il en ressort que, pour les médias, le lancement de ce système de transport intermodal représenta la cristallisation des relations de confiance et de partenariat existant désormais entre les deux nations, apportant la preuve irréfutable que la rivalité entre la France et l'Angleterre qui avait perduré depuis l'époque napoléonienne n'était plus qu'un mauvais souvenir. Sous leur plume, en effectuant d'une traite la liaison entre Londres et Paris, le train-ferry devenait l'incarnation même d'une communauté imaginaire englobant les deux nations.

26- Voir dans le Times les annonces publiées en date du 19 juin, du 8, 10 juillet et du 20 septembre 1936 au sujet de la nouvelle liaison train-ferry entre Dunkerque et Douvres.

27- « Le nouveau train-ferry - mise en service de la liaison Douvres-Dunkerque », The Times, 13 octobre 1936.

28- « Le nouveau service de ferry-boats... », art. cité.

29- The Times, 14 octobre 1936. 
Par la suite, le train-ferry est devenu l'une des images emblématiques caractérisant la France aux yeux du monde. Lors de la Foire internationale de New York, en 1939, l'État français a parrainé l'édition d'un catalogue haut de gamme schématisant l'impact mondial de la France dans les milieux industriel et culturel. L'un des éléments clés de ce catalogue était une très belle carte de la France où chaque province était représentée par un symbole culturel caractéristique $^{30}$. Pour Dunkerque, le choix s'est porté tout naturellement sur le train-ferry, c'est-à-dire l'image d'un petit bateau franchissant la Manche avec, à son bord, un train. Le choix de cette image et sa présence sur la carte constituent une forme de consécration pour cette initiative intermodale qui faisait désormais partie de l'héritage culturel français et comptait parmi les symboles de l'identité nationale.

Sur l'une des affiches publicitaires destinées à promouvoir le train-ferry, le train est présenté comme le symbole du voyage heureux (fig. 1 et fig. 2 ) $^{31}$. L'artiste britannique, auteur de cette affiche, a choisi de dessiner l'image d'une femme plongée dans son sommeil, un sourire aux lèvres. On notera l'interaction intéressante entre les deux images ainsi juxtaposées, le train-ferry évoquant la notion du rêve réalisé. Le visage de la femme qui sourit est devenu, par la suite, l'une des images fortes du nouveau train-ferry. Les affiches en question existaient parallèlement en français et en anglais et ont paru dans la presse française et britannique.

Également consacré à cette initiative intermodale, un article publié dans L'Illustration mettait l'accent sur les nouvelles normes de service et de convivialité offertes aux Européens grâce au ferry-boat ${ }^{32}$. Le texte de cet article était assorti de dessins mettant côte à côte le voyage Londres-Paris avant et après le lancement du nouveau service. Avant 1936, les voyageurs étaient obligés de courir la valise à la main pour monter à bord d'un ferry transmanche. Après 1936, ils pouvaient dormir tranquillement dans leur cabine. L'article paru dans L'Illustration cherchait par ailleurs à rassurer les passagers qui pouvaient avoir des doutes quant à la fiabilité de la liaison entre le ferry et le train.

Le port de Cherbourg accueillit, à son tour, un autre projet intermodal novateur. Cherbourg était l'un des ports français les plus fréquentés, terminus de nombreux paquebots en provenance de New York. Avant la construction du nouveau port, les passagers et les marchandises arrivant à Cherbourg par

30- France: New York World Fair, 1939 [La France, Foire internationale de New York 1939].

31- Voir l'annonce pour le train-ferry Douvres-Dunkerque publiée dans The Times, $1^{\text {er }}$ octobre 1936.

32- Voir «Le Nouveau service de ferry-boats... », art. cité. 


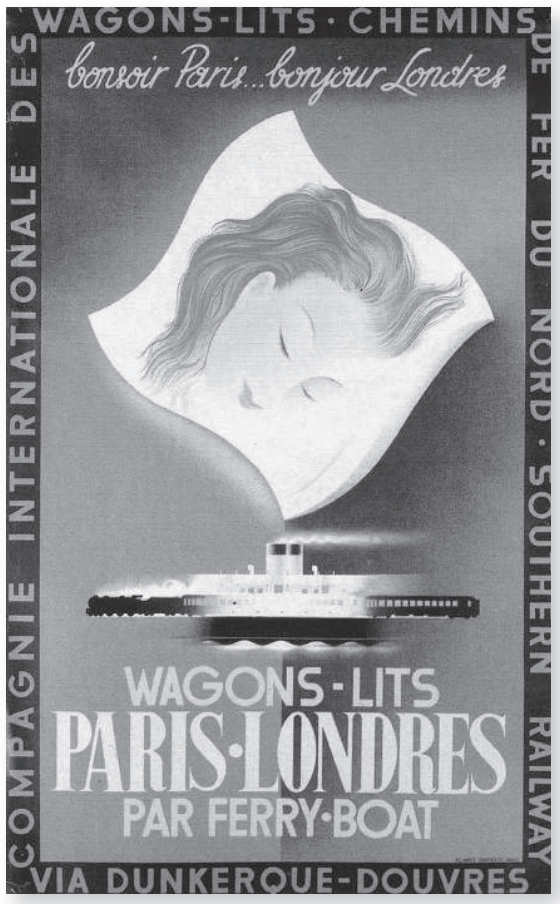

Figure 2. Le train-ferry Douvres-Dunkerque publiée dans The Times, $1^{\text {er }}$ octobre 1936.

Figure 1.

Garett, "Bonsoir Paris, Bonjour Londres.

Wagons-Lits Paris-Londres par ferry-boats", affiche pour la Compagnie du Nord / Southern Railways, Alliance Graphique imprimeur, 1936. CAH SNCF, cote VDR 1059.

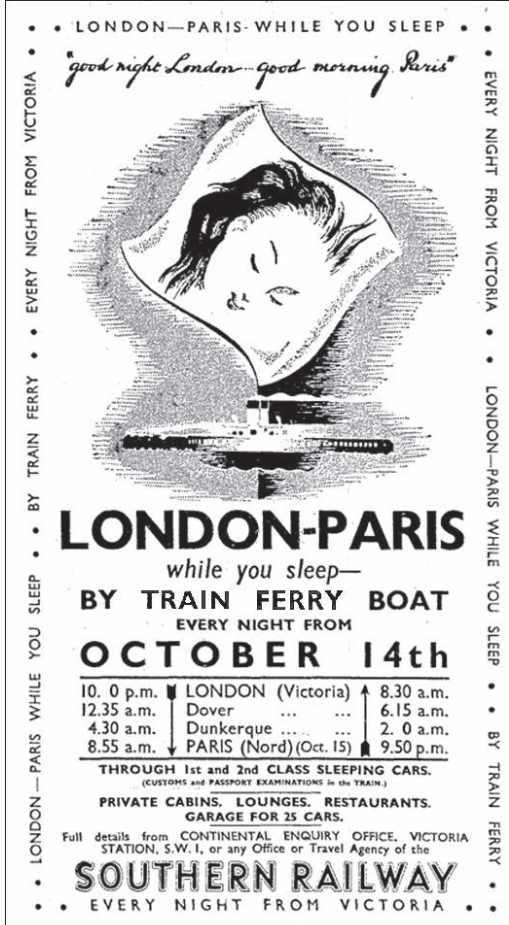


voie maritime devaient emprunter des navettes pour gagner la terre, transbordement onéreux et chronophage. Le $1^{\text {er }}$ mai 1933 a lieu l'inauguration du nouveau port de Cherbourg. Celui-ci comportait des structures facilitant la communication entre mer et terre notamment un système de rails reliant les navires et les trains, comme le montrent les images du port (fig. 3 et fig. 4). Grâce aux nouvelles installations, les passagers pouvaient désormais embarquer directement à bord des transatlantiques. De surcroît, la nouvelle jetée qui s'étendait loin dans la mer pouvait accueillir deux paquebots à la fois.

Le nouveau port de Cherbourg, avec ses installations favorisant l'intermodalité, était remarquable à maints égards. En premier lieu, il a été largement salué comme étant un bel exemple de mise en œuvre de la philosophie du voyage ininterrompu. De nombreux articles à son sujet ont été publiés dans la presse, y compris la presse de masse comme par exemple L'Illustration ou le Times. Dans le cas de L'Illustration, sous la plume de R. Lest le nouveau port est devenu une innovation intermodale porteuse d'une amélioration considérable dans le service offert aux passager ${ }^{33}$. Le port nouvelle mouture jouissait d'une liaison directe avec la gare ferroviaire. En outre, la présence de grues permettait le déchargement rapide des voitures automobiles et les propriétaires de voitures arrivant à bord des paquebots pouvaient désormais repartir par la route dès leur arrivée à Cherbourg : une voie routière reliait le port à la route nationale. Avant la construction du nouveau port, de nombreux bateaux transatlantiques connaissaient des retards importants à leur arrivée à New York, le chargement des navires devant s'effectuer par navette, procédé pénible alors incontournable. Dans le cas où un paquebot arrivait tardivement à Cherbourg, les passagers étaient dans l'obligation de passer une journée supplémentaire à son bord en attendant la réouverture des services d'immigration et de douane. La construction du nouveau port a donc facilité et rendu plus agréables les voyages entre la France et les États-Unis.

Le nouveau port de Cherbourg est vite devenu l'un des symboles d'une France en évolution, d'un pays maritime à l'influence intercontinentale croissante. Au plan esthétique, le nouveau port et, avant tout, sa jetée qui se prolongeait loin vers l'océan, ont su capter l'imagination du peuple français, qui voyait en lui une manifestation du design contemporain. Dans son œuvre Métier d'homme, Raoul Dautry a mis en évidence le rôle joué par les ports en symbolisant l'influence exercée par la France à travers le monde ${ }^{34}$. Dans un texte hautement poétique, Dautry affirma que les ports de la côte occidentale

33- LEST, «L'extension du port de Cherbourg », L'Illustration, 11 mars 1933.

34- DAUTRY, 1937. 


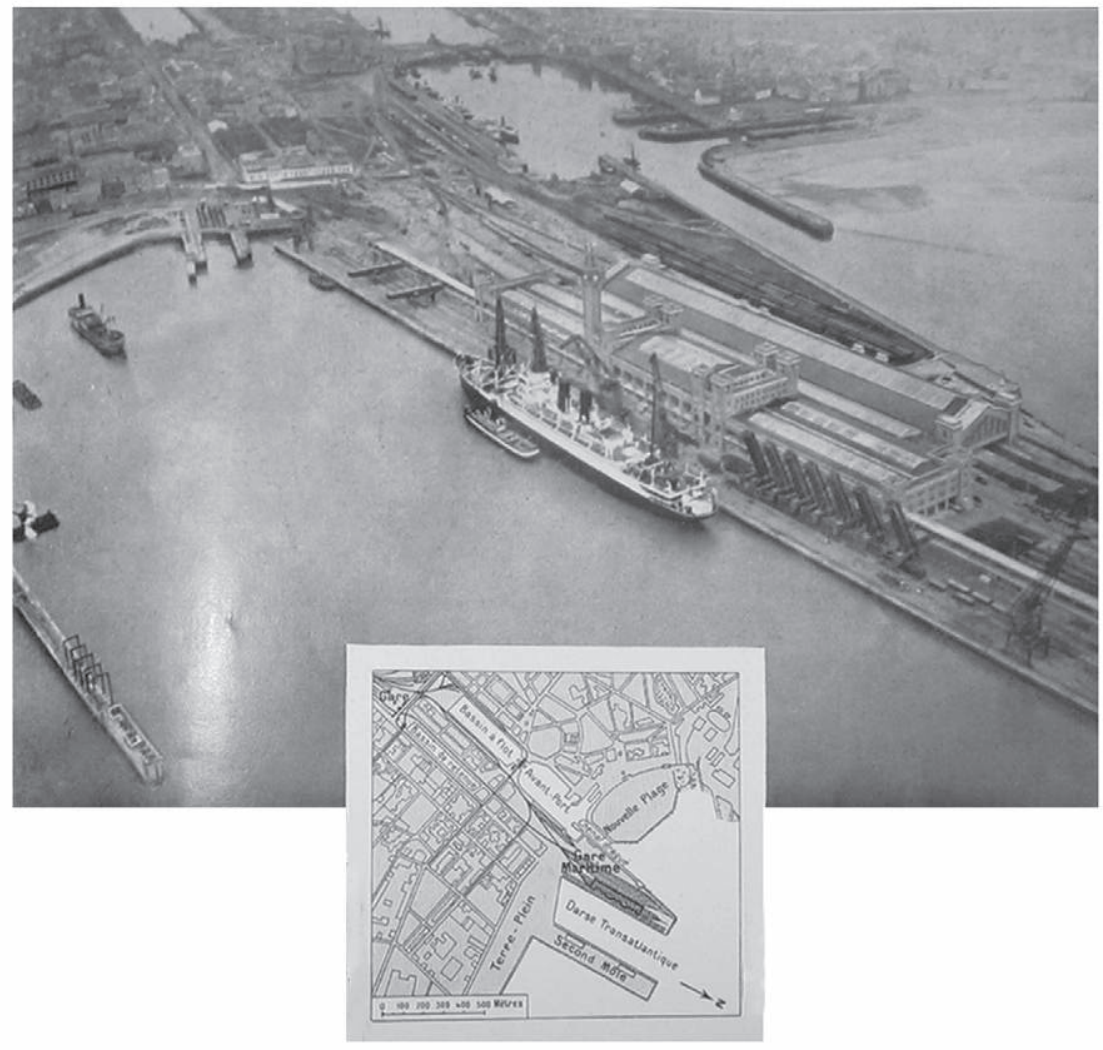

Figure 3 et figure 4 .

R. Lest, «L'extension du port de Cherbourg », L'Illustration, 11 mars 1933.

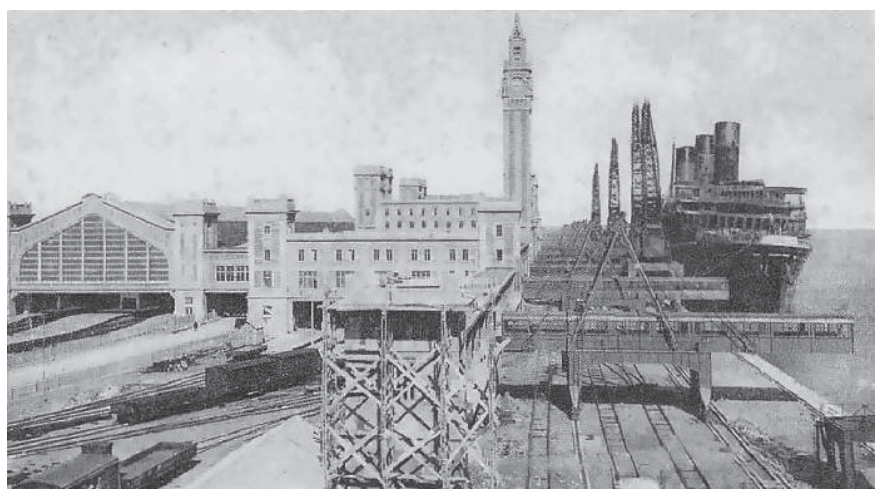


étaient parmi les plus beaux de France. On doit aussi à Dautry une très jolie métaphore sur les ports : "C'est le domaine de notre Réseau qui, à l'extrême pointe de l'Europe, enfoncé dans l'Océan comme une proue de navire, s'allonge vers le Nouveau Monde, se rapproche de l'Angleterre; cueille au sud le trafic africain et celui de l'Amérique du Sud ${ }^{35}$. " Conçu pour tenir compte des exigences de l'intermodalité, le nouveau port incarnait aussi le prestige de la France, nation maritime de premier rang offrant un accès facile au Nouveau Monde. La représentation médiatique des initiatives intermodales était donc marquée par un langage séduisant et des métaphores percutantes qui ont su accrocher l'imagination populaire.

La montée en puissance des réseaux de transport intermodal a également favorisé une amélioration radicale du confort voyageur. Dans leurs publicités, les compagnies de chemins de fer ne manquaient pas de souligner le confort inégalé dont jouissaient leurs clients grâce à la philosophie intermodale. Raoul Dautry, ingénieur en chef des Chemins de fer du Nord et, plus tard, directeur général des Chemins de fer de l'État, disait toujours, avec la passion qu'on lui connait, que l'objet premier des chemins de fer français était celui d'améliorer la qualité des services offerts aux voyageurs. Adepte de la philosophie de l'intermodalité, Dautry lança de nombreux projets visant à faciliter le transfert vers les bateaux des passagers arrivant par le rail dans les ports du littoral occidental. En 1929, Dautry avait lancé le train express Manche-Océan assurant la correspondance à Dieppe avec les bateaux en partance vers Newhaven ${ }^{36}$. Il a également amélioré les correspondances dans d'autres ports. En adaptant l'horaire du Manche-Océan pour mieux correspondre au départ de l'un des ferries, il réussit à réduire de plusieurs heures le temps de trajet des voyageurs empruntant le $\operatorname{train}^{37}$. Dautry fut enfin l'artisan de la mise en service d'un train supplémentaire, pouvant accueillir jusqu’à 646 voyageurs, entre Paris et Cherbourg $^{38}$. Ses nombreux efforts pour assurer la coordination des transports ferroviaire et maritime ont permis de jeter les bases nécessaires au développement des réseaux intermodaux. Une affiche destinée à promouvoir cette initiative ne visait pas seulement à renseigner les passagers sur les améliorations apportées à leur voyage mais également à figurer en un espace unique et continu aussi bien Londres que la France, l'Algérie et l'Espagne (fig. 5). De telles images invitaient la population française à concevoir l'Europe comme un ensemble cohérent.

35- Ibid., p. 207.

36- Voir « Chemins de fer de l'État \& Southern Railway », CAH, 148.BCO 49.

37- DAUTRY, 1937, p. 229.

38- Conseil de Réseau des chemins de fer de l'État. Janvier 1935, séance 2, Archives Nationales, 307 AP 72. 
Elles présentaient l'intermodalité comme l'essence même du voyage moderne, rapide, sans interruption, fluide. Les affiches laissaient entendre que le chemin de fer figurait parmi les acteurs incontournables du renforcement des liens de solidarité entre les différents pays européens.

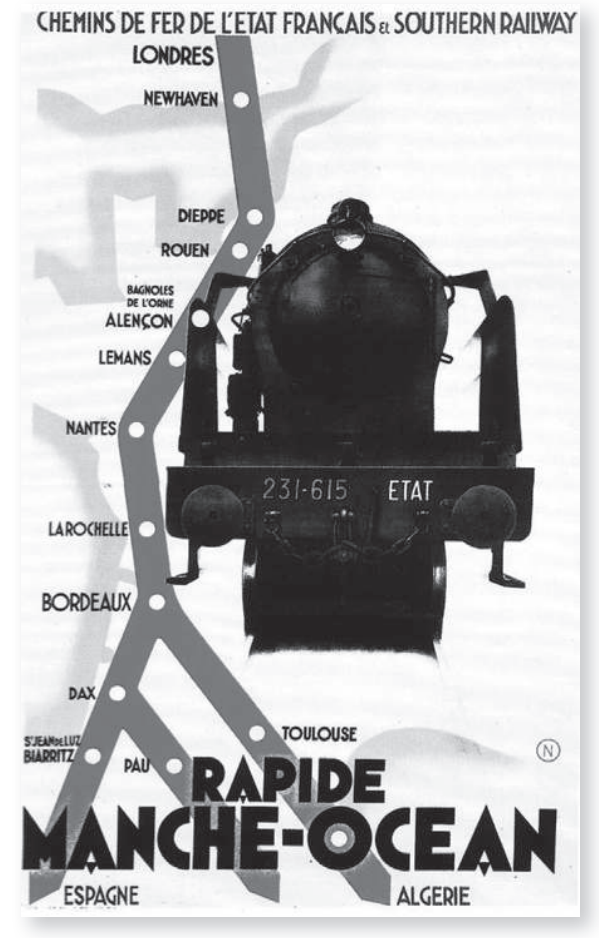

Figure 5 .

Carre, Einholtz et Petit, "Rapide

Manche Océan", affiche pour le Réseau de l'État et Southern Raillway, 1932. CAH SNCF, cote VDR 2024.

La promotion des initiatives intermodales par les médias a joué un rôle majeur dans le succès du transport intermodal en la France durant l'entredeux-guerres. On présentait les projets intermodaux comme les vecteurs de la nouvelle culture de mobilité. Certains thèmes ont marqué la promotion de ces projets, par exemple celui du luxe et du confort dans le cas du Train Bleu, celui de l'amitié entre la France et l'Angleterre dans le cas du train-ferry entre Douvres et Dunkerque, et celui de la France en tant que puissance maritime dans le cas du port de Cherbourg. Les annonces publicitaires, affiches, articles et autres photos publiés par la presse en la matière mettaient en relief la modernité et le confort de ces nouvelles formes de voyage désormais à la portée du peuple français, et ceci grâce à l'intermodalité. 


\section{Bibliographie}

BACHET, BALSO, BÉJUI, et al., I 999 : Bachet, Olivier, Balso, Raoul, Bejui, Pascal, et al., Le Patrimoine de la SNCF et des chemins de fer français, Paris, coll. Le patrimoine des institutions économiques, Flohic, 1999, 971 p., 2 vol.

BEHREND, 1977 : Behrend, George, Histoire des trains de luxe, de l'OrientExpress au T.E.E., Office du livre, Fribourg, 1977, 216 p.

BEHREND, KELLY, 1969 : Behrend, George, Kelly, Vincent, Yatakli Vagon: Turkish Steam Travel, Londres, Neville Spearman Ltd., 1969, 259 p.

CAMPBELL, 1983 : Campbell, Derek, 1883-The Orient Express-1983, Bognor Regis, New Horizon, 1983, 184 p.

CIWL, 1926 : Cinquantenaire de la Compagnie Internationale des Wagons-Lits \& des Grands Express Européens, 1876-1926, Paris, CIWL, 1926, 46 p.

COMMAULT, 1966 : Commault, Roger, Georges Nagelmackers, un pionnier du confort sur rail, Uzès, Éditions de la Capitelle, 1966, 62 p.

COOKRIDGE, 1978 : Cookridge, E.H., Orient Express, the Life and Times of the World's Most Famous Train, New York, Random House, 1978, X-288 p.

CRONIN, 1994 : Cronin, Vincent, Paris. City of Light, 1919-1939, Londres, Harper Collins, 1994, XVII-334 p.

COUDERT, KNEPPER, TOUSSIROT, 2009 : Coudert, Gérard, Knepper, Maurice, Toussirot, Pierre-Yves, La Compagnie des Wagons-Lits : Histoire des vébicules ferroviaires de luxe, Paris, Éditions La Vie du Rail, 2009, 399 p.

DAUTRY, 1937 : Dautry, Raoul, Métier d'homme, Paris, Plon, 1937, 331 p.

DES CARS, COMMAULT, 1976 : Des Cars, Jean, Commault, Roger, Sleeping Story : l'épopée des wagons-lits, Paris, Julliard, 1976, 244 p.

DES CARS, CARACALLA, 1984 : Des Cars, Jean, Caracalla, Jean-Paul, L'Orient-Express : un siècle d'aventures ferroviaires, Paris, Denoël, 1984, $158 \mathrm{p}$.

DES CARS, CARACALLA, 1988 : Des Cars, Jean, Caracalla, Jean-Paul, Le Train Bleu et les grands express de la Riviera, Paris, Denoël, 1988, 159 p.

FUMEY, VARLET, ZEMBRI (sous la dir.), 2009 : Fumey, Gilles, Varlet, Jean, Zembri, Pierre (sous la direction de), Mobilités contemporaines : approches géoculturelles des transports, Paris, Ellipses Édition Marketing SA, 2009, 285 p.

GREGORY, 1991 : Gregory, Alexis, The Golden Age of Travel, 1880-1939, New York, Rizzoli, 1991, 220 p.

GUITARD-AUVISTE 1981 : Guitard-Auviste, Ginette, Paul Morand, 18881976 : légende et vérités, Paris, Hachette, coll. Littérature générale, 1981, 420 p. 
LATRASSE, 1936 : Latrasse (ingénieur en chef adjoint de l'exploitation de la Compagnie du chemin de fer du Nord), "Le service de ferry-boats entre la France et l'Angleterre via Dunkerque et Douvres ", Revue générale des chemins de fer, t. LVI (1936), octobre, p. 232-241.

MORAND, 1994 : Morand, Paul, Le Voyage, Monaco, Éditions du Rocher, $1994,149 \mathrm{p}$.

RODAKOWSKI, 1905 : Rodakowski (de), Ernest, "The Channel Ferry »: advantages and feasibility of a Train-Ferry between England and France with plans and tables, Londres, Harrison \& Sons, 1905, xv-236 p.

SHERWOOD, 1985 : Sherwood, Shirley, Venise-Simplon-Orient-Express, Londres, Wendefeld \& Nicolson, 1985, 160 p.

STAROSTINA, 2007 : Starostina, Natalia, "Engineering the Empire of Images: The Representations of Railways in Interwar France ", thèse de doctorat d'histoire, Emory University, 2007, sous la dir. de Kathryn Amdur, 440 p.

WIESENTHAL, 1979 : Wiesenthal, Mauricio, La Belle Époque de l'OrientExpress, Barcelone, Geocolor, 1979, 94 p. 
